\title{
High Performance Layered Inorganic Flame Retardants: a Review
}

\author{
Dongqin Su, Zehua Tang, Jinfeng Xie, Zhengxu Bian, Junhao Zhang* and Aihua Yuan* \\ School of Environmental and Chemical Engineering, Jiangsu University of Science and Technology, \\ Zhenjiang, Jiangsu 212003, China
}

Email: jhzhang6@just.edu.cn (J. Z.)

\begin{abstract}
Layered inorganic compounds are potential flame retardant materials with good flame retardant performance. In particular, inorganic composites or inorganic-organic hybrids may be a promising candidate of flame retardants. This review introduces the thermal stability, flame retardancy, smoke suppression and their mechanism of layered inorganic-based flame retardants. The results indicate that the incorporation of layered inorganic based flame retardants can improve the thermal stability and residual yield at high temperature, flame retardancy and smoke suppression. The improved flame retardancy and smoke suppression performances were mainly ascribed to layered inorganic based flame retardants with excellent lamellar barrier effect and outstanding catalytic carbonization performance, which was propitious to form compact and stiff carbonaceous ceramic layer, and suppressed efficiently the heat and mass transmission between polymer nanocomposites and flame zone.
\end{abstract}

Keywords layered inorganic compounds, thermal stability, flame retardants, mechanism

\section{Introduction}

With the rapid expansion of polymer science and engineer, polymer-based materials (e.g., polyolefin, epoxy resin, etc.) are widely applied in various fields such as in electric/electronic devices, construction materials, furniture, vehicles, etc. ${ }^{[1-4]}$ However, such wide application brings serious fire hazard caused by polymer's flammability, which is a serious threat to human safety and restricts their applications significantly. ${ }^{[5-8]}$

In order to decrease the flammability of polymer, introducing flame retardant (FR) into polymer is the most convenient and economic technique. Halogen-containing FR had been regarded as an efficient flame retardant and used widely in the industrials due to their outstanding flame retardancy, low-cost and low loading. Nevertheless, it released usually toxic and corrosive gases during combustion that threaten air and water ecosystems. ${ }^{[9,10]}$ Therefore, there is an immediate need to replace them with more environmentally compatible alternatives. With the rapid development of nanotechnology, nanomaterials as catalysts and physical barrier agents are used as efficient and environment-friendly flame retardants. Among them, polymer/layered inorganic nanocomposites including montmorillonite (MMT), layered double hydroxides (LDHs) and carbon based materials, have been extensively investigated to improve the thermal stability and flame retardancy of polymers. ${ }^{[11-13]}$ Polymer nanocomposites have shown great improvements in flame retardant properties as well as other behaviors even at low filler contents, because nanomaterials as catalysts and sheet separating agents can convert the flammable polymer to graphite under fire conditions, acting as a physical barrier and protecting the underlying materials from the action of heat and flame.

Herein, we reviewed the thermal stability, flame retardancy, smoke suppression and their mechanism of layered inorganic-based flame retardants.

\section{Improving Fire Safety of Polymer Nanocom- posites by Layered Inorganic Flame Retardants}

\section{Improving fire safety by modified montmorillonite}

Montmorillonite is phyllosilicates, in which the individual layers are composed of a tetrahedral $(\mathrm{T})$ and an octahedral $(\mathrm{O})$ sheet in either a 1:1 or 2:1 proportion, as shown in Figure 1. Due to isomorphous substitution (e.g., $\mathrm{Al}^{3+}$ for $\mathrm{Si}^{4+}$ in the $\mathrm{T}$ sheet and/or $\mathrm{Al}^{3+}$ for $\mathrm{Mg}^{2+}$ in the $\mathrm{O}$ sheet), the layers of many 2:1 type clay minerals carry a permanent negative charge, which is balanced by cationic counter ions occupying interlayer space. ${ }^{[14]}$ The counter ions can be exchanged by other cations, making MMT expand the interlayer spacing, which is propitious to intercalate polymer chains into the interlayers. When special cations were exchanged into the interlayers, the modified MMT can be further used for improving flame retardancy. Besides, other approaches have been applied to modify the structure and physicochemical properties of MMT. ${ }^{[15]}$

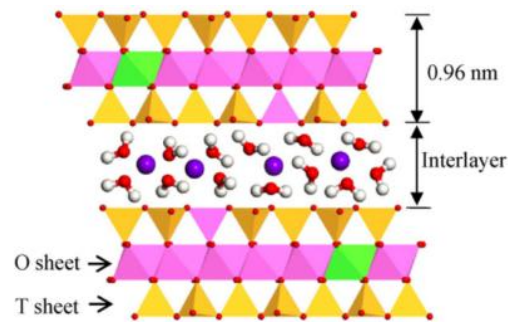

Figure 1 The structure of montmorillonite.

Implanting layered montmorillonite into polymers is known to modify dramatically various physical properties including thermal stability and fire resistance due to catalytic carbonization and lamellar barrier. ${ }^{[16]} \mathrm{A}$ great deal of attention has been focused on the combustion behavior of polymer/MMT nanocomposites. ${ }^{[17,18]}$ Compared with virgin polymer, the MMT nanocomposites decompose at significantly higher temperatures and demonstrate a substantial decrease in the release of 


\section{Minireview}

heat and smoke during combustion.

Previous research results indicated that it's an effective strategy to combine transition metal compounds with intumescent flame retardant (IFR) or carbonization catalyst for reinforcing the network in polymeric matrix. Song et al. ${ }^{[19]}$ reported that modified CNTs by IFR improved flame retardant and mechanical properties of polypropylene due to better dispersion of CNTs and dense char layer. Tang et al. ${ }^{[20]}$ illuminated that the combination of CNTs with $\mathrm{Ni}_{2} \mathrm{O}_{3}$ improved the flame retardant properties of polyethylene based on better dispersion of CNTs and charring by products catalyzed by $\mathrm{Ni}_{2} \mathrm{O}_{3}$. The fillers with different geometrical dimensions can construct three dimensional interconnected network structures in the polymer. ${ }^{[21,22]}$ Inspired by the effectiveness of iron and montmorillonite, Fe-montmorillonite (Fe-MMT) was prepared by simple hydrothermal method. ${ }^{[23]}$ The Fe-MMT has the same crystal structure and the $\mathrm{Fe}^{3+}$ replaces $\mathrm{Al}^{3+}$ in the crystal lattice comparing to Na-MMT. Fe-MMT is modified by cetyltrimethyl ammonium bromide (CTAB) to obtain organic modification Fe-MMT (Fe-OMT), which was incorporated into polymer matrix to prepare a series of Fe-OMT based polymer nanocomposites. ${ }^{[24-27]}$ The LOI and the UL-94 vertical burning test of PP and its nanocomposites indicated that flammability properties of nanocomposites were significantly improved compared to pure PP. The LOls of PP/45wt\%ATH/5wt\%Fe-OMT and PP/ $45 w t \% A T H / 5 w t \% N a-O M T$ were 27 and 23.5 , respectively. It was notable that the dripping phenomenon was eliminated in the PP/ATH/Fe-OMT nanocomposites. However, PP/ATH/ Na-OMT nanocomposites did drip. When the content of Fe-OMT reached $7 w t \%$, UL-94 achieved a V-0 grade. The effect of Fe-OMT in flame-retardancy of PP is superior to that of $\mathrm{Na}-\mathrm{OMT}$, which indicated that Fe-OMT had a better synergistic effect on the flame-retardancy of PP/ATH. ${ }^{[28]}$

MMT possesses nanometer size and layer barrier effects, and iron compounds have significant catalytic carbonization performance. Based on these above, the new montmorillonite intercalation compounds (MICs) were designed and synthesized. ${ }^{[29,30]}$ The iron ions were introduced into MMT interlayers by ion exchange and were transferred to iron compounds nanoparticles to obtain MMT intercalation iron compounds (MIIC) by a redox process using MMT as a reactor. MIIC was modified by CTAB to obtain organic MIIC (OMIIC), which was incorporated into PP/ATH system to prepare PP/ATH/OMIIC nanocomposites. The TG results further prove that OMIIC can enhance the thermal stability of PP/IFR composites at higher temperature and played a key role in promoting the formation of protective char layer. The small amount (5 mass\%) of OMIIC makes PP composites reach $\mathrm{V}-0$ rating and also increases $\mathrm{LOI}$ value to 29.0, as shown in Figure 2. From the results of cone calorimeter tests, the small amount of OMIIC has significant effect on the reduction in the values of heat release rate peak ( $p H R R$ ), total heat release (THR), and smoke production release (SPR). Based on the analysis of morphology of residues after cone calorimeter test, the improving flame retardancy by OMIIC is attributed to the intercalation and exfoliation of the layered OMIIC in the composite matrix that restricts the diffusion of oxygen and decomposed organic volatiles, and iron compound can promote charring of PP. The above results illustrate that there is a synergistic effect between OMIIC and ATH in PP matrix. ${ }^{[31]}$

\section{Improving fire safety by hydroxides or double hydroxides}

Metal hydroxides are the largest commercially manufactured flame retardants and have been widely used in wire and cable insulation products, synthetic marble and onyx, latex for carpet back-coatings, phenolic and epoxy resins, and unsaturated polyesters. ${ }^{[32]}$ The endothermic decomposition process of (a)
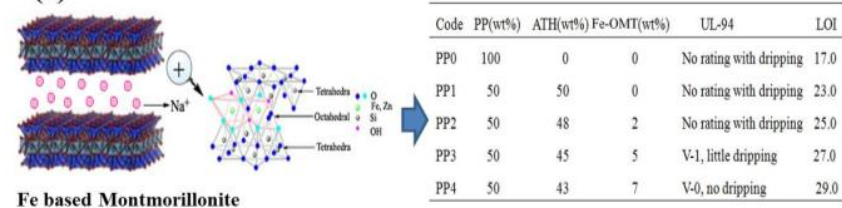

Fe based Montmorillonit

(b)

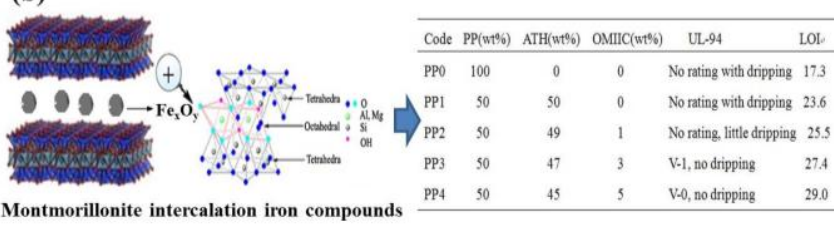

Figure 2 (a) The schematic diagram of Fe-OMT and flame retardancy of PP/ATH/Fe-OMT nanocomposites; (b) The schematic diagram of OMIIT and flame retardancy of PP/ATH/ OMIIT nanocomposites.

magnesium hydroxide $(\mathrm{MH})$ and aluminum hydroxide $(\mathrm{ATH})$ can decrease the temperature of the burning material and release water into the gas phase to dilute the flame. ${ }^{[33]}$ In addition, the presence of anhydrous alumina can help acid-catalyzed dehydration of some polymers and consequently enhance char formation. Since both anhydrous alumina $\left(\mathrm{Al}_{2} \mathrm{O}_{3}\right)$ and magnesia $(\mathrm{MgO})$ are white and highly refractory powders, they provide heat insulation by reflecting heat when they accumulate on a surface. ${ }^{[34]}$ The main disadvantage of $\mathrm{MH}$ and $\mathrm{ATH}$ is their low flame retardant efficiency, which requires high loadings combined with their inherent poor compatibility. As a result, addition of large amounts of either $\mathrm{MH}$ or ATH leads to severe deterioration of the mechanical properties of polymers. ${ }^{[35]}$ In Figure 3 , ultrathin $\beta-\mathrm{Co}(\mathrm{OH})_{2}$ nanosheets with the thickness of about 2.6 $\mathrm{nm}$ were successfully synthesized, which exhibited excellent flame retardancy and smoke suppression properties in TPU composites. Compared with pure TPU, the addition of $4 \mathrm{wt} \%$ ultrathin $\beta-\mathrm{Co}(\mathrm{OH})_{2}$ nanosheets had over $52 \%$ reduction in
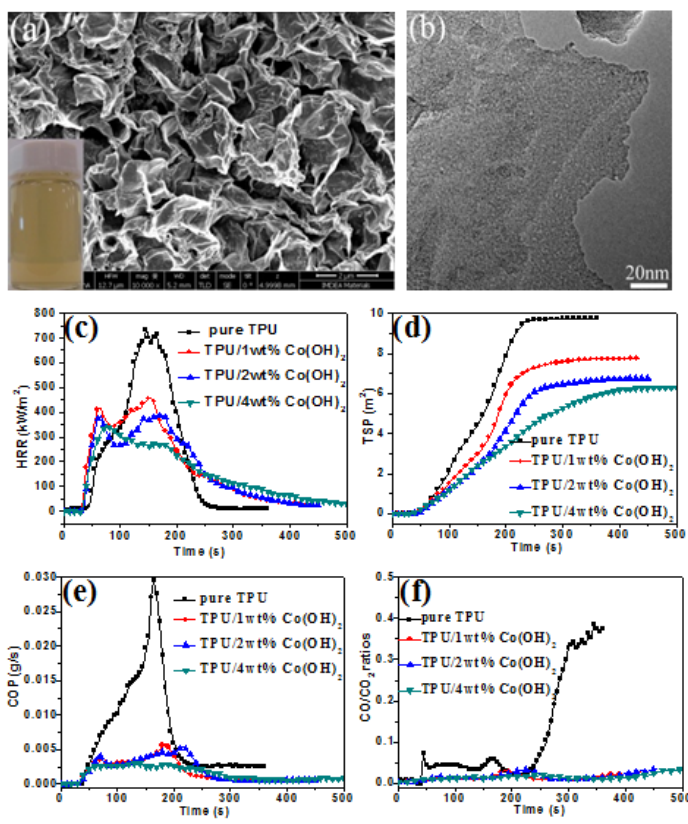

Figure 3 (a) SEM image of ultrathin $\beta-\mathrm{Co}(\mathrm{OH})_{2}$ nanosheets and the inset; (b) TEM image of a typical ultrathin $\beta-\mathrm{Co}(\mathrm{OH})_{2}$ nanosheet; (c) HRR curves of TPU/Co(OH) 2 nanocomposites; (d) Total smoke release of TPU/Co(OH) $)_{2}$ nanocomposites; (e) $\mathrm{CO}$ production (COP); (f) $\mathrm{CO} / \mathrm{CO}_{2}$ ratios. 
pHRR. The results of smoke release showed that the maximum intensity of the evolved $\mathrm{CO}$ reduced $89.7 \%$, the $\mathrm{CO} / \mathrm{CO}_{2}$ ratios of $\mathrm{TPU} / \mathrm{Co}(\mathrm{OH})_{2}$ nanocomposites were reduced significantly after $235 \mathrm{~s}$, and TSP decreased about $35.7 \% .{ }^{[36]}$

$\mathrm{LDHs}$ are a class of anionic lamellar compounds made up of positively charged brucite-like layers with an interlayer region containing charge compensating anions and molecules of solvation. ${ }^{[37]}$ Most LDHs have a general molecular formula of $\left[M_{1-x}{ }^{2+} M_{x}^{3+}(\mathrm{OH})_{2}\right]^{x+} \mathrm{A}^{n} / x^{n-} \cdot \mathrm{zH}_{2} \mathrm{O}$, where $\mathrm{M}^{2+}$ and $\mathrm{M}^{3+}$ are divalent and trivalent metal cations, such as $\mathrm{Mg}^{2+}, \mathrm{Al}^{3+}$, respectively. $\mathrm{A}^{\mathrm{n}-}$ is an intercalated inorganic or organic anion, such as $\mathrm{CO}_{3}{ }^{2-}$, $\mathrm{NO}_{3}^{-}, \mathrm{SO}_{4}{ }^{2-}, \mathrm{RCO}_{2}^{-}$, and $x$ is normally between $0.2-0.4$. Occasionally a monovalent or tetravalent cation may be substituted for the divalent or trivalent ions respectively, such as $\mathrm{Li}^{+}$or $\mathrm{Ti}^{4+}$. Figure 4 shows a schematic representation of hydrotalcite structure. The excellent flame retardancy and smoke suppression properties of LDHs are derived from their unique chemical composition and layered structure. Just like cationic clays, LDHs can be modified by intercalating organic anions into the interlamella galleries. These organic-modified LDHs can be used as nanofillers for the synthesis of polymer-LDH nanocomposites. ${ }^{[38]}$ Although no mechanism has been proposed for the flame retardant mechanism of LDH nanocomposites, it is generally believed that the flame retardant mechanism of LDHs is different from that of silicate clay-based nanocomposites. During thermal decomposition, LDHs will lose the interlayer $\mathrm{H}_{2} \mathrm{O}$, decomposition of the intercalated anions (e.g., $\mathrm{CO}_{3}{ }^{2-}$ ) and the metal hydroxide network will produce $\mathrm{H}_{2} \mathrm{O}$ and other gases (e.g., $\mathrm{CO}_{2}$ ). The effects for these processes will reduce fuel available for combustion, which will lower heat release and eventually stop the combustion when there is not enough fuel to propagate the reaction, and promote the formation of an expanded carbonaceous coating or char. Char formation protects the bulk polymer from exposure to air thus reducing the heat release during the combustion and suppressing smoke production. ${ }^{[39]}$ LDHs, as flame-retardant fillers alone, not only have good flame retardant properties, but also exhibit excellent flame retardancy when used in conjunction with other flame retardants. ${ }^{[40,41]} \mathrm{NiAl-LDH}$ was synthesized via co-precipitation and modified by sodium dodecyl sulfate, marked as NiAl-OLDH. The results indicated that incorporation of NiAl-OLDH in PP/IFR composites improved significantly the thermal stability, flame retardancy and smoke suppression properties. With the addition of 5 wt\% NiAl-OLDH, PP/IFR/ $\mathrm{NiAl}-\mathrm{OLDH}$ nanocomposites obtained UL-94 V-0 level and the LOI value reached $27.5 \%$. Compared with those of PP/IFR, the pHRR, THR and pSPR values reduced $49 \%, 10 \%$, and $63 \%$, respectively, which were attributed to the barrier effect and excellent charring performance of $\mathrm{NiAl-OLDH.}{ }^{[42]}$

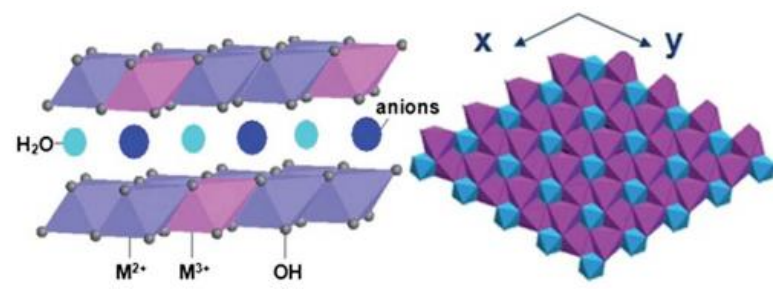

Figure 4 Schematic structure of layered double hydroxides.

\section{Improving fire safety by carbon based materisals}

Up to now, carbon-based materials include graphite, graphene, CNT, fullerene, etc. These materials are not isolated from each other. For example, graphene, the latest member in this family, is an atomically thick, two-dimensional (2-D) sheet (Figure 5a), regarded as the building block of all other graphitic carbon allotropes with different dimensionality: 3-D graphite is made of graphene sheets stacked on top of each other with a spacing distance of $3.37 \AA$ (Figure 5b); 1-D CNT may be made by rolling graphene sheets (Figure $5 \mathrm{c}$ ); $0-\mathrm{D}$ fullerenes (buckyballs) may be envisioned to be made by wrapping a part of graphene (Figure 5d). Graphene, the building block of all graphitic forms, can be wrapped up into 0-D buckyballs, rolled into 1-D nanotubes, or stacked into 3-D graphite. Due to their extraordinary intrinsic properties and ability to be dispersed in various polymer matrices, carbon-based materials have been widely used in fabricating polymer composites/nanocomposites in order to improve the mechanical, thermal, gas barrier, conductivity, and flame retardant performances.
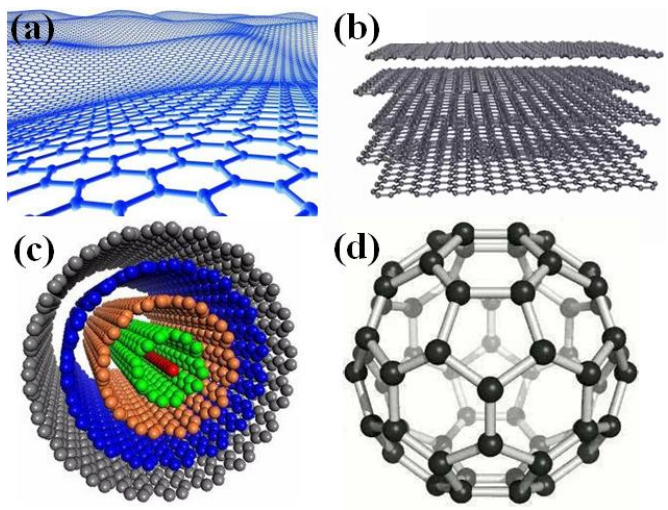

Figure 5 (a) graphene; (b) graphite; (c) carbon nanotubes; (d) fullerene.

Fullerene is an allotrope of carbon with spheroidal shaped structure. The incorporation of fullerene into a polymer matrix is an important technological accomplishment as it allows combining excellent thermal stability and good physical strength of fullerenes with the well-known advantages of polymers. For instance, PP/fullerene nanocomposites have been prepared via melt compounding, and the results from cone calorimetry suggest that the flame retardant property of PP/fullerene nanocomposites is improved with increasing the loading level of fullerene. The results showed that the pHRR of PP/fullerene nanocomposite was reduced by $46 \%$ compared to that of neat PP at the fullerene loading of $2 \mathrm{wt} \%{ }^{[43]}$ In addition to pristine fullerene, the application of functionalized or hybrid fullerenes in flame retardant polymer materials has been investigated. A dendrimer-like fullerene decorated oligomeric intumescent flame retardant $\left(\mathrm{C}_{60}\right.$-d-PDBPP) has been synthesized. The resulting $\mathrm{PP} / \mathrm{C}_{60}$-dPDBPP nanocomposites show significantly improved thermal oxidation resistance and reduced flammability compared to those of pure polymer. ${ }^{[4]}$ Furthermore, fullerene decorated carbon nanotubes $\left(\mathrm{C}_{60}-\mathrm{d}\right.$-CNTs) were fabricated and applied in flame retarding PP. ${ }^{[4]}$ The pHRR observed from cone calorimetry is reduced by nearly $71 \%$ by the presence of only $1 \mathrm{wt} \%$ of $\mathrm{C}_{60}$-d-CNTs. Compared to pristine fullerenes, $\mathrm{C}_{60}$-d-CNTs confer better flame retardancy to PP because of the cooperative interaction between the radical-trapping effect of the fullerene and the barrier effect of the CNT network.

CNTs are a promising alternative to the use of conventional flame retardants. Adding a small amount (usually $<5 \mathrm{wt} \%$ ) of CNTs has been reported to reduce the fire hazards for a wide range of polymers. ${ }^{[46]} \mathrm{PP} / \mathrm{CNTs}$ nanocomposites have been prepared by melt blending without using any organic treatment or additional additives. The addition of CNTs imparted high fire retardant efficiency in PP: The pHRR of the nanocomposites with 1 vol\% CNTs is reduced by about $73 \%$ compared to that of PP. ${ }^{[47]}$ Polymer/CNTs nanocomposites exhibit low flammability in terms of heat release rate but it fails in other burning meas- 
urements, particularly LOI and UL-94 tests. Hence, CNTs are combined with other flame retardant additives in order to create a synergistic effect. In our previous work, a novel type of Fe-CNTs was prepared, which was used as a nanofiller to improve efficiently catalytic carbonization, flame retardancy and mechanical performances of epoxy (EP). With the incorporation of $6 \mathrm{wt} \% \mathrm{Fe}-\mathrm{CNTs}$, the LOI value of EP/Fe-CNTs nanocomposites was improved to 35.0 , and UL-94 V-1 rating was passed with the average $\left(t_{1}+t_{2}\right)$ value of 13.3. Compared with pure EP, the pHRR, THR and TSP of EP/6wt\%Fe-CNTs nanocomposites were decreased $30.7 \%, 39.1 \%$ and $48.6 \%$, respectively. The significantly improved flame retardancy could be attributed to the compact carbonaceous ceramic layers, which retarded the transfer of heat and combustible gas between EP nanocomposites and flame zone. ${ }^{[48]}$
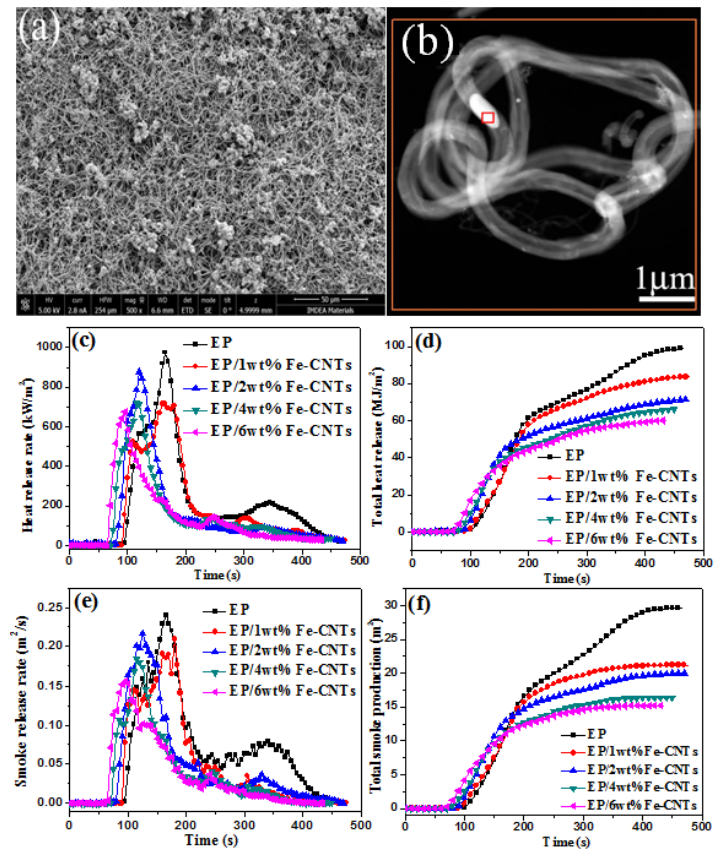

Figure 6 (a) SEM image of Fe-CNTs; (b) STEM image of Fe-CNTs; (c)-(f) HRR, THR, SPR, TSP curves of pure EP and EP/Fe-CNTs nanocomposites.

Due to its good intrinsic flame retardant property, pristine graphene has been utilized directly to prepare flame retardant polymer composites. In contrast to other nano- and micro-sized carbon fillers such as expanded graphite, nano-scaled carbon black and multiwall carbon nanotubes, ${ }^{[49]}$ only graphene affords uniform dispersion combined with simultaneously improved stiffness, electrical conductivity and enhanced flame retardancy of PP, as evidenced by the reduced pHRR (-76\%). Graphene/ $\mathrm{PU}$ nanocomposites prepared from solution blending method show a good flame retardant and smoke suppression behaviors with a significant reduction in total smoke release $(25 \%$ reduction with 1 wt\% graphene) and a lower pHRR and THR. ${ }^{[50]}$ In our previous report, graphene oxide (GO) nanocoating was deposited on the surface of polyurethane sponge (PUS) to reduce its flammability via layer-by-layer (LBL) assembly method. This coating system eliminated completely melt dripping of sponge upon exposure to the direct flame from a butane torch, which also exhibited excellent flame retardancy. ${ }^{\text {[11] }}$

Although organic flame retardants functionalized graphene ${ }^{[52]}$ and inorganic/graphene hybrids ${ }^{[53]}$ have been demonstrated to be effective in lowering the HRR of polymers, most of these composites fail in LOI and UL-94 tests. In order to meet industrial requirement, graphene has been used as a synergist for flame retarding polymers in combination with a wide variety of conventional flame retardants. Striking multiple synergies created by combining graphene and CNTs have been observed: with $0.5 \mathrm{wt} \%$ of graphene and $0.5 \mathrm{wt} \%$ of CNTs. The pHRR value of PP composites was decreased by $73 \%$, which was superior over its counterparts with $1.0 \mathrm{wt} \%$ of graphene or CNTs. ${ }^{[54]}$ As aforementioned, the optimal flame retardancy may be a balance between the thermal conductivity and the barrier effect of graphene that is similar to that of CNTs. Recently, thermally insulating and fire-retardant lightweight anisotropic foams have been prepared based on nanocellulose and GO, together with sepiolite nanorods (SEP). ${ }^{[55]}$ UL-94 vertical burning tests show that nanocellulose-based composite foams with an optimized addition of GO (10 wt\%) and SEP (10 wt\%) display very good fire retardancy, where the flame does not self-propagate. Foams with suboptimal composition, that is, with no GO or low SEP content, exhibit some fire retardancy, but they shrink much more than the nanocomposite foams with optimal composition, indicating the existence of synergism between GO and SEP. The combination of graphene with other flame retardants also exhibits excellent anti-dripping properties during the combustion of polymers. For instance, a UL-94 V-0 PBS composite with excellent anti-dripping behavior is obtained for a formulation of $18 \mathrm{wt} \%$ IFR and $2 \mathrm{wt} \%$ graphene. ${ }^{[56]}$ Melt flow index measurement indicates that the presence of grapheme enhances significantly the melt viscosity and thus restrains the melt dripping.

Pristine graphite is rarely used in flame retardant polymers. The carbon flakes in natural graphite stack so compactly that the penetration of the resin matrix into the slots of the graphite sheets is very difficult. Instead, expandable graphite (EG) prepared from natural graphite has been widely used as a flame retardant for a wide range of polymers due to its easy exfoliation in a polymer matrix. It is convenient to blend EG with polymers since $E G$ is a commercially available fire retardant. PA6/EG and PA6/clay composites have been prepared by a melt-compounding method, and EG shows significant reductions in pHRR ( $60 \%$ compared to neat polyamide 6$)$ and MLR, slightly superior over that obtained for organoclay composites. ${ }^{[57]}$ Although EG is an inexpensive and abundant flame retardant additive, it has obvious disadvantages: one is high loading required for a satisfactory flame retardant level that leads to a deleterious effect on the mechanical properties of polymer matrix, and the other is the thermally unstable char layers formed during combustion. Therefore, EG needs to be combined with other flame retardants to achieve a high flame retardant efficiency. A number of studies have been conducted on EG filled flame retardant polymers, giving satisfactory fire resistance for some polymers. ${ }^{[58-62]}$ Due to the volume expansion nature of $E G$ during combustion, $E G$ has been widely combined with phosphorus-containing compounds to form intumescent flame retardant system for polymers, where an intumescent and stable char layer could be generated.

\section{Conclusions}

Layered inorganic materials have been widely used in fabricating polymer nanocomposites over the past few decades. In contrast to conventional flame retardants, the incorporation of layered inorganic materials can improve the fire retardancy of polymers as well as the mechanical properties, thermal stability, promising behavior for the develop high performance polymer nanocomposites. The general principles of the action of layered inorganic materials as flame retardants do not significantly differ from the conventional retardants. There are two main routes, in which the burning process can be retarded: (1) The addition of layered inorganic materials is beneficial to the formation of a 
protective layer, which can shield the underlying materials against flame and meanwhile slow down the "fuel flow" to feed the flame. (2) Some layered inorganic materials such as fullerenes and CNTs can absorb highly active radicals, so that the chain reaction of combustion process is interrupted.

\section{References}

[1] Mittal, V. Manufacturing of Nanocomposites with Engineering Plastics, Woodhead Publishing, 2015, pp. 15-29.

[2] Soares, B. G. J. Mol. Liq. 2018, 262, 8.

[3] Hu, Z.; Shao, Q.; Xu, X. R.; Zhang, D. Y.; Huang, Y. D. Compos. Sci. Technol. 2017, 142, 294.

[4] Hu, Z.; Shao, Q.; Moloney, M. G.; Xu, X. R.; Zhang, D. Y.; Li, J.; Zhang, C. H.; Huang, Y. D. Macromolecules 2017, 50, 1422.

[5] Wicklein, B.; Kocjan, A.; Salazar-Alvarez, G.; Carosio, F.; Camino, G.; Antonietti, M.; Bergstrom, L. Nat. Nanotechnol. 2014, 10, 277.

[6] Liu, Y.; Gao, Y. S.; Wang, Q.; Lin, W. R. Dalton Trans. 2018, 334, 108.

[7] Lim, K. S.; Li, Z. W.; Li, X. H.; Zhang, Z. J. Chem. Eng. J. 2017, 58, 227.

[8] Hirschler, M. M. Fire Mater. 2015, 3, 232.

[9] Ravichandran, S.; Nagarajan, S.; Ku, B. C.; Coughlin, B.; Emrick, T.; Kumar, J.; Nagarajan, R. Green Chem. 2012, 14, 819.

[10] Meinier, R.; Sonnier, R.; Zavaleta, P.; Suard, S.; Ferry, L. J. Hazard. Mater. 2018, 342, 306.

[11] Wang, J. N.; Su, X. Y.; Mao, Z. P. Polym. Degrad. Stab. 2014, 109, 154.

[12] Liu, Y.; Gao, Y. S.; Wang, Q.; LIn, W. R. Dalton Trans. 2018, 47, 14827.

[13] Kausar, A.; Rafique, I.; Muhammad, B. Polym. Plast. Technol. Eng. 2017, 56, 470.

[14] Zhu, R. L.; Chen, Q. Z.; Zhou, Q.; Xi, Y. F.; Zhu, J. X.; He, H. P. Appl. Clay Sci. 2016, 123, 239.

[15] Yan, L.; Xu, Z. S.; Wang, X. H. Prog. Org. Coat. 2018, 122, 107.

[16] Lopez-Cuesta, J. M. Clay-Polymer Nanocomposites, Elsevier, 2017, pp. 443-473.

[17] Wang, J. N.; Mao, Z. P. J. Appl. Polym. Sci. 2014, 131, 39625.

[18] Ren, X. N.; Zong, R. W.; Hu, Y.; Lo, S. M. Polym. Compos. 2018, 39, 1234

[19] Song, P. G.; Xu, L. H.; Guo, Z. H.; Zhang, Y.; Fang, Z. P. J. Mater. Chem. 2008, 18, 5083.

[20] Yu, H. O.; Liu, J.; Wang, Z.; Jiang, Z. W.; Tang, T. J. Phys. Chem. C 2009, 113, 13092

[21] Lee, S. K.; Bai, B. C.; Im, J. S.; In, S. J.; Lee, Y. S. J. Ind. Eng. Chem. 2010, 16, 891.

[22] Isitman, N. A.; Kaynak, C. Polym. Degrad. Stab. 2010, 95, 1523.

[23] Kong, Q. H.; Hu, Y.; Yang, L.; Fan, W. C. Polym. Compos. 2006, 27, 49.

[24] Liu, H.; Zhong, Q.; Kong, Q. H.; Zhang, X. G.; Li, Y. J.; Zhang, J. H. J. Therm. Anal. Calorim. 2014, 117, 693.

[25] Kong, Q. H.; Wu, H.; Zhang, H. K.; Zhang, X. G.; Zhao, W. L.; Zhang, J. H. J. Nanosci. Nanotechnol. 2016, 16, 8287.

[26] Liu, H.; Kong, Q. H.; Cheng, Y. H.; Cao, G. Y. Chin. J. Chem. 2012, 30, 1594.

[27] Kong, Q. H.; Hu, Y.; Song, L.; Tang, Y. W. J. Therm. Anal. Calorim. 2011, 104, 1145

[28] Kong, Q. H.; Hu, Y.; Song, L.; Yi, C. W. Polym. Adv. Technol. 2009, 20, 404.

[29] Kong, Q. H.; Zhang, H. K.; Zheng, L.; Wang, D. Y.; Zhang, J. H. Polym. Adv. Technol. 2017, 28, 965.

[30] Kong, Q. H.; Wu, T.; Zhang, H. K.; Zhang, Y.; Zhang, M. M.; Si, T.
Y.; Yang, L.; Zhang, J. H. Appl. Clay Sci. 2017, 146, 230.

[31] Liu, L.; Zhang, H. K.; Sun, L.; Kong, Q. H.; Zhang, J. H. J. Therm. Anal. Calorim. 2016, 117, 693.

[32] Gao, Y. S.; Wu, J. W.; Wang, Q.; Wilkie, C. A.; O'Hare, D. J. Mater. Chem. $A$ 2014, 2, 10996.

[33] Feng, Y.; Tang, P.; Xi, J.; Jiang Y.; Li, D. Recent Pat. Nanotechnol. 2012, 6, 231.

[34] Nodera, A.; Kanai, T. J. Appl. Polym. Sci. 2004, 94, 2131.

[35] Rothon, R.; Hornsby, P. Polym. Degrad. Stab. 1996, 54, 383.

[36] Zhang, J. H.; Kong, Q. H.; Yang, L. W.; Wang, D. Y. Green Chem. 2016, 18, 3066.

[37] Wang, Q.; O'Hare, D. Chem. Rev. 2012, 112, 4124.

[38] Leroux, F.; Besse, J. P. Chem. Mater. 2001, 13, 3507.

[39] Guo, B. T.; Liu, Y. Z.; Zhang, Q.; Wang, F. Q.; Wang, Q. W.; Liu, Y. X.; Li. J.; Yu, H. P. ACS Appl. Mater. Interfaces 2017, 9, 23039.

[40] Wu, T.; Kong, Q. H.; Zhang, H. K.; Zhang, J. H. J. Nanosci. Nanotechnol. 2018, 18, 1051.

[41] Zheng, L.; Wu, T.; Kong, Q. H.; Zhang, J. H.; Liu, H. J. Therm. Anal. Calorim. 2017, 129, 1039.

[42] Kong, Q. H.; Wu, T.; Wang, J. Q.; Liu, H.; Zhang, J. H. J. Nanosci. Nanotechnol. 2018, 18, 3660.

[43] Kuan, C. F.; Chen, W. J.; Li, Y. L.; Chen, C. H.; Kuan, H. C.; Chiang, C. L. J. Phys. Chem. Solids 2010, 71, 539.

[44] Song, P. A.; Liu, H.; Shen, Y.; Du, B.; Fang, Z. P.; Wu, Y. J. Mater. Chem. 2009, 19, 1305

[45] Song, P. A.; Shen, Y.; Du, B.; Guo, Z.; Fang, Z. P. Nanoscale 2009 $1,118$.

[46] Wang, X.; Kalali, E. N.; Wan, J. T.; Wang, D. Y. Prog. Polym. Sci. 2017, 69, 22.

[47] Kashiwagi, T.; Grulke, E.; Hilding, J.; Harris, R.; Awad, W.; Douglas, J. Macromol. Rapid Commun. 2002, 23, 761.

[48] Zhang, J. H.; Kong, Q. H.; Wang, D. Y. J. Mater. Chem. A 2018, 6, 6376.

[49] Dittrich, B.; Wartig, K. A.; Hofmann, R.; Mülhaupt, R.; Schartel, B. Polym. Degrad. Stab. 2013, 98, 1495.

[50] Hu, J.; Zhang, F. J. Therm. Anal. Calorim. 2014, 118, 1561.

[51] Kong, Q. H.; Wu, T.; Liu, H.; Zhang, Y.; Zhang, M. M.; Cai, Y. B.; Zhang, J. H.; Yang, L. J. Nanosci. Nanotechnol. 2018, 18, 5105.

[52] Jiang, S. D.; Bai, Z. M.; Tang, G.; Hu, Y.; Song, L. Compos. Sci. Technol. 2014, 102, 51.

[53] Wang, D.; Zhang, Q. J.; Zhou, K. Q.; Yang, W.; Hu, Y.; Gong, X. L. J. Hazard. Mater. 2014, 278, 391.

[54] Song, P. A.; Liu, L.; Fu, S.; Yu, Y.; Jin, C.; Wu, Q.; Zhang, Y.; Li, Q. Nanotechnology 2013, 24, 1

[55] Wicklein, B.; Kocjan, A.; Salazar-Alvarez, G.; Carosio, F.; Camino, G.; Antonietti, M.; Bergstron, L. Nat. Nanotechnol. 2015, 10, 277.

[56] Wang, X.; Song, L.; Yang, H. Y.; Lu, H. D.; Hu, Y. Ind. Eng. Chem. Res. 2011, 50, 5376.

[57] Dasari, A.; Yu, Z. Z.; Mai, Y. W.; Cai, G.; Song, H. Polymer 2009, 50, 1577.

[58] Feng, F.; Qian, L. Polym. Compos. 2014, 35, 301.

[59] Bai, G.; Guo, C.; Li, L. Constr. Build. Mater. 2014, 50, 148.

[60] Alongi, J.; Frache, A.; Gioffredi, E. Fire Mater. 2011, 35, 383.

[61] Zheng, J.; Li, B.; Guo, C.; Wu, Q.; Wang, Y. J. Appl. Polym. Sci. 2014, 131, 376.

[62] Wu, G. M.; Schartel, B; Bahr, H.; Kleemeier, M.; Yu, D.; Hartwig, A. Combust. Flame 2012, 159, 3616.

Received August 12, 2018 Accepted November 22, 2018 